\title{
Design of Construction Stability Test System using Small Unmanned Aerial Vehicle based on Image Processing
}

\author{
Sung-suk Choi*, Eung-kon Kim* \\ * Department of Computer Science, Sunchon National University, Korea \\ mstar2000@sunchon.ac.kr, kek@sunchon.ac.kr \\ Corresponding Author : Eung-kon Kim
}

\begin{abstract}
Stability test of high-rise buildings must be achieved periodically for the buildings' maintenance, but there are many problems that it is dangerous for inspector to make a personal test, it needs much time and cost, and accurate diagnosis is difficult.

This paper proposes a monitoring system for construction stability test based on image processing to analyze and process data from sensors in the vehicle to inspect internal and exterior crack and deformation of constructions by using unmanned aerial vehicle. The system loads various sensors such as temperature sensor, humidity sensor, smoke sensor, illuminance sensor, CO2 sensor, ultrasonic sensor, and infrared thermal imaging sensor and can achieve stability inspection. So, It can reduce inspection time and building's maintenance cost.
\end{abstract}

Keywords - Small Unmanned Aerial Vehicle, Monitoring Facilities, Safety Inspection, Image Processing, Sensor Fusion

\section{INTRODUCTION}

Recently, the accidents by damage and collapse of construction facilities increase while it takes lots of time and costs and is very dangerous for the inspector to inspect such large facilities of high rise buildings directly with the equipment. Such direct inspection is very dangerous and takes lots of time to examine the multiple numbers of structures that a correct diagnosis is difficult. So, the inspection method using an unmanned aerial vehicle is urgently needed for checking the place where the access of inspector is limited, integral inspection of multiple numbers of structures and checking the damage according to seasonal and daily time difference. Unmanned aerial vehicle (UAV) is a flight system where the pilot is not boarded but is controlled remotely by the person on the ground and called as a drone which means the bee's buzz. It was mostly used for a target of the surveillance or military use previously but now is used for various uses including the private and research survey [1]

As IT industry develops rapidly, it is recently converged with other industries. It became a generalization that the computer handles the works which are difficult and hard to be done by people grafting IT onto construction field.
They implement the architectural drawings by 3D, simulate the building in advance or calculate the more accurate data in advance by estimation and measurement using the computer for correct survey and maintenance when construct the bridges or bridge piers. In addition, even the technology of augmented reality is used in order to provide more vivid sense of reality and immersion.

Currently, the structural safety inspection is tried in various methods by grafting IT.

In this paper, a structural safety inspection system based on augmented reality using a small unmanned aerial vehicle is designed. The proposed system offers an image recognition monitoring system through the sensor data wireless transmission by mounting multiple sensors for a small aerial vehicle platform based structural monitoring.

This system has an advantage that they could provide a more accurate and precise inspection and diagnosis through the image processing on the ground by understanding the problem after accessing the high rise structure through remote control mounting an imaging camera on the small aerial vehicle.

\section{RELATED RESEARCHES.}

In Korea, the continuous safety inspection and management for the facilities is required as the number of decrepit high rise structures more than 20 years since built increases. Such decrepit structures become a big risk factor when they are damaged by poor management, and the collapse accident occurred in industrial zone could be a typical case. Since it is extremely difficult and dangerous for the inspector to access the high rise building higher than several tens of meters or $100 \mathrm{~m}$, the equipment to make a safe and fast inspection is needed. The wired measuring and testing methods require high costs for installation as well as replacement and repair when it gets an error. Thus, the collection of images and sensor data using a small aerial vehicle which may shorten the safety inspection time and save the maintenance cost, transmission technology and data analysis skill are required.

Now, many studies on the control technology by unmanned aerial vehicle are carried out in order to apply it to documentary filming or crop dusting [2], and the technology to substi- 
tute the physical sensors to monitor the fire or motion is developed to a significant level for image analysis technology [3].

Especially, Japan, Germany and UK showed high interests in the maintenance and safety inspection of the high rise structures that Fraunhofer Institute in Germany and Cyberhawk Co., Ltd in UK developed and commercialized the small aerial vehicle to manage the safety of wind power structures.

The small aerial vehicle developed by Cyberhawk in UK may find the cracks and deformation of the rotor blade installed at the height of approx. 100m through remote control but it may detect the external cracks bigger than $6 \mathrm{~mm}$ only because it uses the low-resolution image [4].

The infrared thermography system developed by NEC in Japan may exactly check the structural problems which could not be checked visually such as delamination and crack of external wall of the structure, but its application is limited to low-rise construction structures.

\section{SYSTEM EXPLANATION}

This paper proposes the development of a system which may detect the internal and external defects of high-rise facilities and inspect other various points using aerial vehicle based various sensors, high resolution camera and thermo image camera by supplementing the disadvantages of existing systems.

\section{A. Hardware and the whole system configuration}

The whole configuration diagram of the system proposed in this paper is shown on Figure.1.

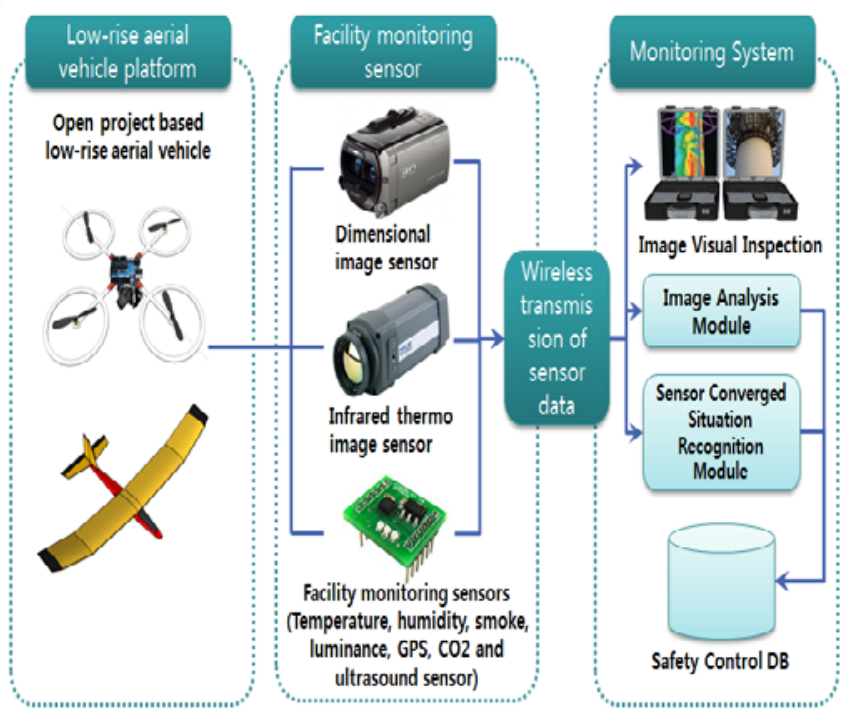

Figure 1. System Configuration Diagram

The open source based aerial vehicle based on Microcopter in Germany shall be used in order to mount multiple sensors to conduct the safety inspection of the structure.

Microcopter developed aerial vehicles such as Qudrocopter (4 blades), Hexacopter (6 blades) and Octocopter (8 blades) according to number of propeller blades [5]. They are suitable as the basic small aerial vehicles for safety inspection of the structure because the flight control devices including the altitude maintaining device (acceleration sensor), level maintaining device (gyro sensor), attitude stabilizing function (pressure sensor) and GPS are mounted on Flight Control Board [6].

Mount an image camera on this small aerial vehicle and provide an image frame of the small aerial vehicle to the tablet PC on the ground through synchronization process and such tablet PC shall make an image processing with each frame input. In addition, it is possible to provide more accurate safety control of the structure by mounting various sensors.

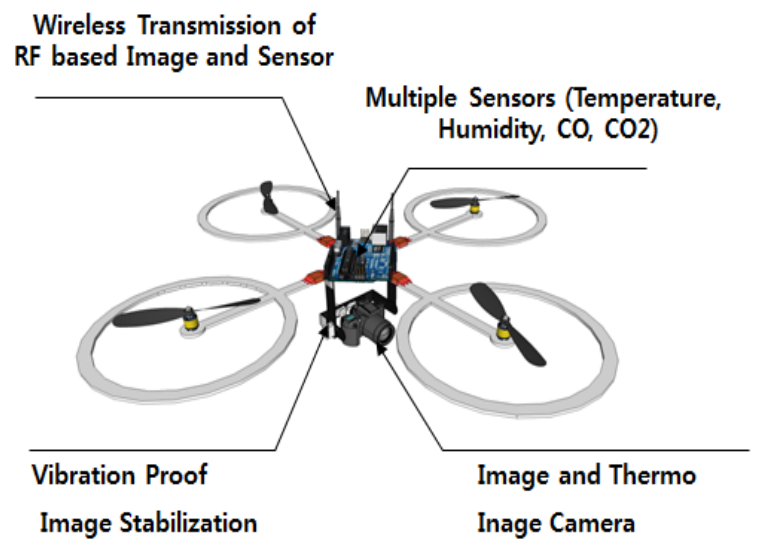

Figure 2. Aerial Vehicle Customizing

Detailed hardware specifications of the small aerial vehicle to mount the multiple sensors are as follows:

TABLE 1. HARDWARE SPECIFICATIONS OF THE SMALL AERIAL VEHICle

\begin{tabular}{|l|l|}
\hline Item & Specification \\
\hline Diameter & $800 \mathrm{~mm}$ \\
\hline Frame & Aluminium \\
\hline Weight (without battery) & $3 \mathrm{Kg}$ \\
\hline Propeller & 14 inch \\
\hline Motor Thrust & $1.5 \mathrm{Kg} /$ motor \\
\hline Operation Time & Longer than 30 minutes \\
\hline Gimbal & $\begin{array}{l}300 \mathrm{~mm}(\mathrm{~W}) \times 300 \mathrm{~mm}(\mathrm{H}), \\
200 \mathrm{~mm}(\text { Bore })\end{array}$ \\
\hline
\end{tabular}

\section{B. Multiple Sensor Mounting Module and Fusion Technol- ogy Design}

It is dangerous and takes lots of time and cost for the inspector to check the damage on the target area according to seasonal and daily time difference. However, it is possible to manage the structure efficiently reducing such risk factors by inspecting the structure mounting sensors on the small aerial vehicle. Thus, the safety inspection of the structure shall be 
made using various kinds of sensors in this paper. Since the big or heavy sensor modules shall become obstacles for the flight, small and light sensor modules shall be mounted on the small aerial vehicle to provide the safety of the flight.

As seen in Figure 2, a universal sensor mounting module to mount various kinds of sensors such as temperature sensor, humidity sensor, CO sensor, CO2 sensor, Ozone sensor, Dust sensor and VOC (volatile organic compound) sensor could be mounted shall be developed and MCU (main control unit) module to mount such sensors shall be made based on ATMega128.

The role of microcontroller in the sensor device is very important because this module controls the whole system. This function is to adjust the data, sampling rate, acquired time and acquisition method measured in the sensor board module and to transmit such data to wireless modem module. Such function is provided by the program stored in the flash memory built in the microcontroller. ATMega128 of Atmel provides the flash memory of $128 \mathrm{k}$ Bytes and 4,000 commands in total [7].

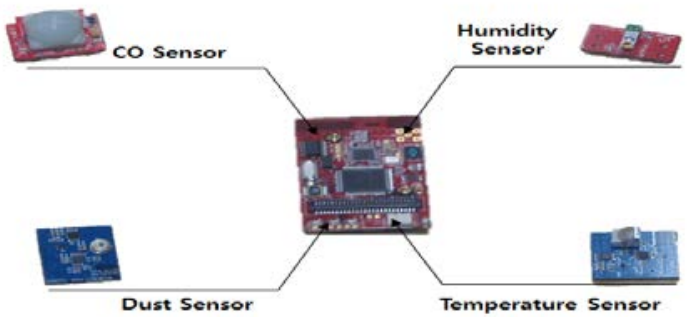

Figure 3. Multiple Sensor Mounting Module

Various sensor fusion methods based on Kalman filter have been continuously studied in order to efficiently use the information obtained from several sensors by fusion.

The sensor fusion methods could be mainly divided to the measurement fusion and the status variable fusion and again could be sub-divided to measurement fusion model, track to track fusion model (TTF), modified track to track fusion model (MTF) and track fusion model with fused prediction (TFP) according to fusion time [8].

Kalman filter was developed by Rudolf Kalman in the early 1960s and is an algorithm which has been often used for computer vision, robot, rocket and satellite and missile control fields [9]. Namely, it is an algorithm used for estimating a new result removing the noise contained in the data using the data measured in the past and the new data [10]. This paper intends to use such Kalman filter for more accurate image processing through estimation of position and attitude control of the small aerial vehicle using such Kalman filter.

\section{Development of Multiple Sensor Data Transmission Technology}

The multiple sensor data collected from the aerial vehicle shall be transmitted to the monitoring personnel on the ground, so a system consisting of IEEE $802.11 \mathrm{n}$ based wireless transmission module and 7dbi antenna shall be configured in order to transmit such data to the place which is at least $100 \mathrm{~m}$.
The sensor data obtained from various sensors such as temperature sensor, humidity sensor, CO sensor, CO2 sensor, Ozone sensor, Dust sensor and VOC (volatile organic compound) shall be visualized to be processed. The meter data managing module which manages the data type, unit and effective area of sensor data shall be developed to process various types of sensor data. The visualization module by each sensor data shall be implemented and displayed on the tablet PC monitor of the worker that the information could be more efficiently managed.

\section{Development of Image Processing Technology to mount Image and Thermo Image Sensor}

The visual inspection is the most basic inspection to conduct the safety inspection of the structure and enhances the accuracy of the safety inspection through more vivid and precise image filming using the high resolution PTZ camera.

Specification of PTZ camera which shall conduct the visual inspection is as follows:

TABLE 2. SPECIFICATION OF PTZ CAMERA

\begin{tabular}{|l|l|}
\hline Item & Specification \\
\hline Image Sensor & $1 / 4^{\prime}$ ' CCD \\
\hline Lens & $4.1 \sim 73.8 \mathrm{~mm}$, F1.3 3.0, auto focus \\
\hline $\begin{array}{l}\text { Viewing angle } \\
\text { of lens }\end{array}$ & $2.7^{\circ} \sim 48^{\circ}$ \\
\hline $\begin{array}{l}\text { Resolution } \\
\text { Zoom }\end{array}$ & $160 \times 120 \sim 704 \times 576$ \\
\hline $\begin{array}{l}\text { Weight } \\
\text { Pumptical, } 12 \mathrm{x} \text { digital zoom }\end{array}$ & Within $800 \mathrm{~g}$ \\
\hline Shooting Speed & 9 fps $~ 30 \mathrm{fps}$ \\
\hline
\end{tabular}

PTZ (Pan/Tilt/Zoom) camera has 2 motors for Pan (horizontal direction) and Tilt (vertical direction) in order to control the image camera mounting module while the zoom function controls the camera lens. Pan function enables the camera to shoot the target area of the structure during flight supporting the fast speed of $100^{\circ}$ per a second within the range of $\pm 170^{\circ}$. Tilt function supports the fast speed of $90^{\circ}$ per a second within the range of $30^{\circ} 90^{\circ}$.

The infrared ray is an electromagnetic wave between the visible ray area and microwave area and its wave area is approx. $0.75 \sim 0.1 \mathrm{~cm}$ and radiated from any object which has heat. The thermo image system is a device which displays the temperature distribution on the surface to thermo image by converting the amount of infrared rays obtained by the infrared camera.

Since the surface of concrete may have a crack by the temperature difference due to different delivery path of thermal 
energy between the sound portion and deformed portion when the thermal energy stored on the ground is radiated from the surface of concrete due to the temperature difference from the air, the thermo image camera shall be used for detecting the thermal energy of concrete surface of internal and external walls of high-rise facilities and for various safety checks such as internal excitation and crack examination in order to detect such cracks in advance.

Specification of small, light and low power thermo image camera to be mounted on the aerial vehicle is as follows:

TABLE 3. SPECIFICATION OF THERMO IMAgE CAMERA

\begin{tabular}{|l|l|}
\hline Item & Specification \\
\hline $\begin{array}{l}\text { Infrared wave } \\
\text { length bandwidth }\end{array}$ & $8 \sim 14 \mu \mathrm{m}$ \\
\hline Measuring Area & $-20^{\circ} \mathrm{C} \sim 250^{\circ} \mathrm{C}$ \\
\hline $\begin{array}{l}\text { Viewing angle of } \\
\text { lens }\end{array}$ & $10.2^{\circ} \times 7.7^{\circ}$ \\
\hline Resolution & $640 \times 480$ pixel \\
\hline Type of sensor & Un-cooled focal plane array \\
\hline Weight & $800 \mathrm{~g}$ \\
\hline Shooting Speed & $9 \mathrm{fps} \sim 30$ fps \\
\hline
\end{tabular}

IEEE 802.1x based wireless transmission technology shall be used in order to transmit the images obtained from the image and thermo image cameras mounted on the aerial vehicle to the worker on the ground. The Cameras shall apply Motion JPEG video compression which may transmit the clear image to each scene and quickly check the safety of the structure by transmitting the images at the speed higher than 20 30fps and resolution of $4 \mathrm{CIF} / 2 \mathrm{CIF}$.

The image processing technology is required in order to process the images of the video. When the images transmitted to the imaging camera and thermo image camera are damaged, not clear or when they want to see the interested area in detail, the data could be managed more effectively using the image processing technology.

\section{E. Development of Image Processing Technology}

The images transmitted from the aerial vehicle may have differences in visibility according to weather or surrounding environment, so it needs to improve the images in accordance with the purpose.

The technologies to improve the image include the smoothing, sharpening and noise rejection etc.

The image analysis is a work to extract the specific area and is often used for the work to find the size, shape and contour line. In addition, following processing steps shall be required in order to recognize the images:

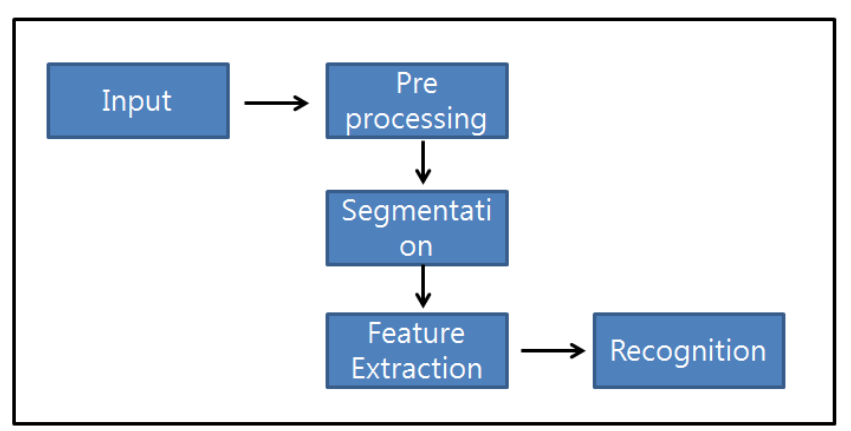

Figure 4. Image Recognition Processing Steps

The images shot from the small aerial vehicle shall go through the pre-processing process, and in this moment the edge detection method which rejects the noise seen on the image shall be applied.

The edge occurs between the objects or between the object and background on the image and refers to the boundary of the object on the image [11]. The detection method using the edge does not require lots of calculation and normally identify the object with the brightness change of surrounding background and its boundary, the edge.

The edge information shall be detected by the 1st differential function such as Prewitt operator, Robert operator and Sobel operator or the 2nd differential function such as Laplacian operator or other many methods [12].

Canny edge detection method which is strong against noise shall be used in this paper.

The image processing process was simply tested using Canny Edge Detection Algorithm as follows:

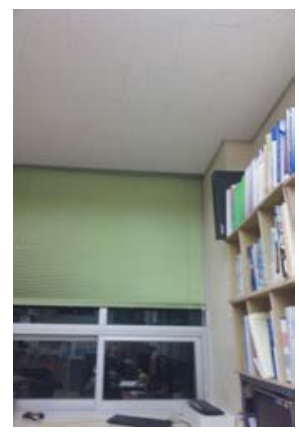

(a) Before Application

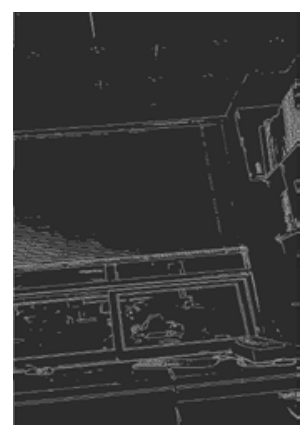

(b) After Application
Figure 5. Canny edge detection algorithm test

The images processed by this algorithm shall use the extraction algorithm through image segmentation in order to improve the processing speed and save the storage capacity. It has an advantage to judge the defect easily comparing with existing images extracting the feature using the segmented images. So, the image processing technology is effective for checking status of the structure and its management.

The features of image were simply extracted using the feature extraction algorithm as follows: 


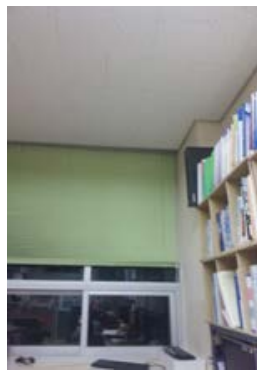

(a) Before Application

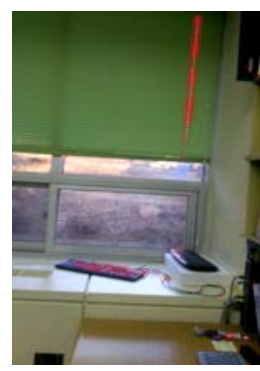

(b) After Application
Figure 6. Feature Extraction Algorithm Test

The basic unit of the image is Pixel, and numerous pixels are gathered to organize an image. Thus a significant loss of time and data could occur when transmission because the quantity of image data is relatively bigger than that of general message data. Since the quantity of images transmitted to image and thermo image camera using the small aerial vehicle is relatively bigger than that of general data, such advantage must be considered.

The image compression technology shall be used for the purpose of eliminating the overlapped or unnecessary data in order to store or transmit the data efficiently. Motion JPEG (M-JPEG) video compression shall be applied for the quick image transmission in this paper.

\section{F. Development of Augmented Reality based Structure Safety Monitoring System}

The augmented reality is a technology to show the virtual objects or information in real time by adding them to the real world, and is normally called as Mixed Reality by mixing the reality and virtual world [13].

The term of Augmented Reality was appeared first time when Tom Caudell assembled the cables of aircraft of Boeing in 1990 based on HMD developed by Ivan Sutherland in 1968 [14].

The augmented reality technology is mainly divided to indoor augmented reality (indoor AR) which is implemented inside building according to characteristic of the space which is implemented and outdoor augmented reality (outdoor AR) implemented outside building.

It is possible for indoor AR to control the factors which affect the accuracy because of the relatively short distance between the target and camera and the space limit. On the other hand, it is difficult for the outdoor AR to estimate the position of the camera due to wide area of space and there are many factors which affect the accuracy. Thus, the outdoor AR decides the position and direction of the camera using GPS and gyro sensor [15].

In this paper, the augmented reality is to be used by mounting the cameras and sensors on the aerial vehicle in order to check the position and status of the structure in the outdoor environment. When the position of the structure is identified, the graphic which marks the information of the virtual objects on the real image shall be loaded and the data shall be visual- ized using the augmented reality so that the worker on the ground could work more easily.

That is, a system which may inspect the safety of the structure comprehensively and check the defects based on the images obtained from the aerial vehicles and the information collected from multiple sensors shall be developed by developing the augmented reality based structure inspection technology which provides the inspector with images obtained from image camera and thermo image camera mounted on the aerial vehicle and multiple sensor data. In addition, the safety inspection of the structure could be conducted conveniently and safely in future through the objective data for safety of the structure and identification of exact position of defect by matching the position, azimuth and altitude of the aerial vehicle and visualize them to the inspector.

Recently, the mobile related augmented reality is developed due to the distribution of Smart phones.

When the users put the built-in camera of the Smart phone to specific object, various 3D models shall be augmented and displayed to provide the users the contents which have more sense of reality.

Since the mobile based augmented reality has a high scalability and is not limited by time and space, it could be implemented at any place and time. Thus, it is now widely used for various fields and is under active study especially in industrial, education and medical fields [16]. The study in future following this paper has a plan to conduct the safety inspection of the structure using the mobile based augmented reality.

\section{IV.CONCLUSION}

This paper proposed a design of safety inspection system of the structure using the small aerial vehicle.

We became to know that the appearance of the structure could be identified by clearer image rejecting the noise using the image processing algorithm for image processing of the images obtained from the aerial vehicle.

It is possible to obtain much more safe and objective inspection process and result comparing to the existing methods by providing more convenient working environment mounting the image camera, thermo image camera and various sensors on the small aerial vehicle

Based on the design in future, it shall be suitable for safety inspection of power line tower, plant stack, bridge and bridge pier and wind power generation facilities which are difficult and dangerous for the people to access.

In case of power line tower, the safety inspection using the aerial vehicle shall be more safe and fast than the direct inspection by climbing the power line by the people.

In case of the plant stack, it shall require lots of cost for conducting the direct inspection by the people after stopping the operation of the plant, but the safety inspection using the aerial vehicle enables the safety inspection of the stack while continuously operating the plant and analyse the gas emitted during the operation.

Besides of this, it could be utilised for the safety inspection, such as detachment or crack inspection for the high-rise build- 
ing, apartments or other concrete structures, and also could be used for exploration using the thermal detection in wide area such as monitoring the forest fire using the thermo image camera mounted on the aerial vehicle.

Therefore, the system proposed in this paper is expected to have a high utilization in the safety inspection in various industries in future.

\section{ACKNOWLEDGMENT}

This research was financially supported by the Ministry of Education (MOE) and National Research Foundation of Korea(NRF) through the Human Resource Training Project for Regional Innovation (No. 2013H1B8A2032217).

\section{REFERENCES}

[1] Hundley, Richard, and Gritton, EugeneC, "Future Technology-Driven Revolutions in Military Operations", RAND Corporation, Document No. DB-110-ARPA, 1994.

[2] Jae-Ha Ryi, Wook Rhee and Jong-Soo Choi. "Effective Perceived Noise Level Prediction for a Propeller driven UAV by using Wind Tunnel Test Data”, Journal of Korean Society for Aeronautical \& Space Science, No. 41(1), pp. 10-16 , Jan. 2013.

[3] Min Goo Yoo and Sung Kyung Hong, “Target Tracking Control of Quadroto UAV using Vision Sensor”, Journal of Korean Society for Aeronautical \& Space Science, No. 40(2), pp. 118-128, 2012.

[4] http://www.thecyberhawk.com/

[5] http://mikrokopter.de/en/home

[6] AscTec Hummingbird with AutoPilot User's Manual, Ascending Technologies GmbH

[7] http://www.atmel.com/devices/atmega128.aspx

[8] C. M. Rogers, "Methods of Multisensor Tracking”, Proceedings of the London Communications Symposium 2003, Communications Engineering Doctorate Centre ISBN: 0-9538863-3-6

[9] Kalman, R. E. 1960. "A New Approach to Linear Filtering and Prediction Problems," Transaction of the ASME-Journal of Basic Engineering, pp. 35-45 (March 1960)

[10] Gan. Q, Harris. C. J, “Comparison of two measurement fusion methods for Kalman-filter-based multisensory data fusion”, Aerospace and Electronic Systems, Vol37, Issue 1, pp.273-279, Jan, 2001.

[11] Hea-jung Lee, Sung-tae Jung, Suck-tae Joung, "Noise Elimination and Edge Detection based on Fuzzy Logic", The journal of the Korea Institute of Maritime Information \& Communication Sciences, Vol7, No3, pp.506512, 2003

[12] S. E. El-Khamy, “A Modified Fuzzy Sobel Edge Detector.”, Radio Science Conference, pp. 1-9, 2000,Feb, 22 24.

[13] Azuma, R.T., "Survey of Augmented Reality", Presence, Teleoperators and Virtual Environments, Vol.6, No.4, Aug. 1997.
[14] P. Milgram and F.Kishino, "A Taxonomy of Mixed Reality Visual Displays, “ IEICE Trans, Information Systems, Vol.E77-D, No.12, pp.1321-1329, 1994.

[15] Seongsu-Jeong, Joon-Heo, Sunkyu-Woo "Development of Building Monitoring Techniques Using Augmented Reality”, Korean Journal of Construction engineering and management, Vol. 10, No. 6, Nov. 2009

[16] Azuma.R, Baillot.Y, Behringer.R, Feiner.S, "Recent advances in augmented reality”, Computer Graphics and Applications, IEEE, Vol.21, Issue 6, pp.34-47, 2001

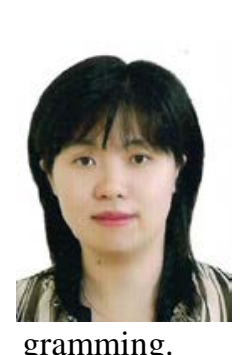

\section{Sung-Suk Choi}

Sung-Suk Choi received the B.S. degree from Korea National Open University, Korea, in 2010. She is currently a M.S student in computer science at the Sunchon National University, Korea, Her current research interests include augmented reality, image processing, computer graphics, android pro-

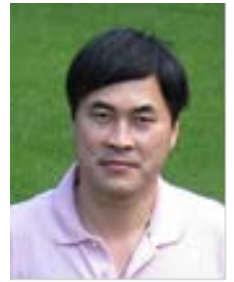

Eung-kon Kim(Corresponding Author) Eung-kon Kim received the B.S. degree from Chosun University, Gwangju,, Korea, in 1980, his M.S degree from department of electronics, Hanyang University, Seoul, Korea, in 1987, his Ph.D. degree from Chosun University, Gwangju, Korea, in 1992. His current research interests are computer vision, virtual/augmented reality, image processing, and computer graphics. Currently he is a professor in department of computer engineering, Sunchon National University, Korea. 